\title{
Solidity in Sequence Spaces
}

\author{
I. J. MADDOX
}

ABSTRACT. Relations are established between several notions of solidity in vectorvalued sequence spaces, and a generalized Köthe-Toeplit\% dual space is introduced in the setting of a Banach algebra.

\section{INTRODUCTION}

The study of linear spaces of scalar sequences and their $\alpha$-duals was initiated by Köthe and Toeplitz [4].

If $s$ denotes the linear space of all infinite sequences $a=\left(a_{k}\right)$ of complex numbers $a_{k}$ and if $E$ is a linear subspace of $s$ then, following [4]; see also [3] and [1], we define the $\alpha$-dual of $E$ as

$$
E^{n}=\left\{a \in s: \sum_{k=1}^{\infty}\left|a_{k} x_{h}\right|<\infty \text { for all } x \in E\right\} \text {. }
$$

Two related dual spaces are defined by

$$
\begin{aligned}
& E^{\beta}=\left\{a \in s: \sum_{k=1}^{\infty} a_{k} x_{k} \text { converges for all } x \in E\right\}, \\
& E^{\gamma}=\left\{a \in s: \sup _{n}\left|\sum_{k=1}^{n} a_{k} \cdot x_{k}\right|<\infty \text { for all } x \in E\right\} .
\end{aligned}
$$

Topologies on a sequence space, involving $\beta$ and $\gamma$ duality have been examined by Garling [2], who noted that $E^{\alpha}=E^{\beta}=E^{\gamma}$ when $E$ is solid (or normal), i.e. when $x \in E$ and $\left|r_{k}\right| \leq\left|x_{k}\right|$ for all $k \in N$ together imply that $! \in E$.

1991 Mathematics Subject Clatsilication: $46 \wedge 45$.

Fditorial de la Universidad Complutense. Madrid. 1991. 
Thus, for example, the space $c_{0}$ of null sequences is solid, but the space $c$ of convergent sequences is not.

We shall be concerned with the more general situation of vector-valued sequences $x=\left(x_{k}\right)=\left(x_{1}, x_{2}, \ldots\right)$ with $x_{k}$ in a complex linear space $X$. By $s(X)$ we denote the linear space of all sequences $x=\left(x_{k}\right)$ with $x_{k} \in X$ and the usual coordinatewise operations: $\alpha x=\left(\alpha x_{k}\right)$ and $x+y=\left(x_{k}+y_{k}\right)$, for each $\alpha \in C$.

If $\lambda=\left(\lambda_{k}\right)$ is a scalar sequence and $x \in s(X)$ then we shall write $\lambda x=\left(\lambda_{k} x_{k}\right)$.

In case $X$ is also a normed space we denote by $B$ the closed unit ball of $X$ and by $B(X)$ the space of all bounded linear operators on $X$. As usual $X^{*}$ denotes the continuous dual space of $X$. Two subspaces of $s(X)$ that we consider later are

$$
\begin{aligned}
& l_{\infty}(X)=\left\{x \in s(X): \sup _{k}\left\|x_{k}\right\|<\infty\right\}, \\
& l_{1}(X)=\left\{x \in s(X): \sum_{k=1}^{\infty}\left\|x_{k}\right\|<\infty\right\} .
\end{aligned}
$$

These spaces generalize the classical spaces $b_{\infty}$ and $/$ which are subspaces of $s$.

If $A=\left(A_{k}\right)$ is a sequence in $B(X)$ we shall write $A x=\left(A_{k} x_{k}\right)$ for each $x \in s(X)$.

Some information about types of generalized Köthe-Toeplitz duals involving sequences of linear operators may be found in Maddox [5].

We now consider the eight statements below, each of which expresses some notion of solidity for a linear subspace $E$ of $s(X)$. It is statement (5) which generalizes the original idea of solid (or normal) as given by Köthe and Toeplit? [4].

The first three statements are meaningful in any complex linear space, but the last five statements require $X$ to be normed. A statement such as $\left\|y_{n}\right\|=\left\|x_{n}\right\|$ is an abbreviation for $\left\|y_{n}\right\|=\left\|x_{n}\right\|$ for all $n \in N$. Also, in (6) to (8) the $A_{n}$ are elements of $B(X)$.

(1) $x \in E$ and $\lambda \in l_{\infty}$ imply $\lambda x \in E$

(2) $x \in E$ and $\left|\lambda_{n}\right| \leq 1$ imply $\lambda x \in E$

(3) $x \in E$ and $\left|\lambda_{y}\right|=1$ imply $\lambda x \in E$

(4) $x \in E$ and $\left\|y_{n}\right\|=\left\|x_{n}\right\|$ imply $y \in E$ 
(5) $x \in E$ and $\left\|y_{n}\right\| \leq\left\|x_{n}\right\|$ imply $y \in E$

(6) $x \in E$ and $\left(\left\|\mathcal{A}_{n}\right\|\right) \in l_{\infty}$ imply $A x \in E$

(7) $x \in E$ and $\left\|A_{n}\right\| \leq 1$ imply $A x \in E$

(8) $x \in E$ and $\left\|A_{n}\right\|=1$ imply $A x \in E$.

\section{EQUIVALENCES}

In Theorems 1, 2 and 3 we determine the relations between the statements (1) to (8).

Theorem 1. In any complex linear space $X$ the statements (1), (2) and (3) are equivalent.

Proof. It is trivial that $(1) \rightarrow(2) \rightarrow(3)$. Let us show that $(3) \rightarrow(1)$. If (3) holds, $x \in E$ and $\lambda \in l_{\infty}$ then there exists $M>0$ with $\left|\lambda_{t t}\right| \leq M$ for all $n \in N$. Define

$$
\mu_{n}=\lambda_{n} / M=\alpha_{n}+i \beta_{n}
$$

where $\alpha_{n}$ and $\beta_{n}$ are real. Then $\left|\alpha_{n}\right| \leq 1$ and $\left|\beta_{n}\right| \leq 1$, so we may choose $\gamma_{n}$ and $\delta_{n}$ with

$$
\alpha_{n}^{2}+\gamma_{n}^{2}=\beta_{n}^{2}+\delta_{n}^{2}=1
$$

Define $z_{n}=\alpha_{n}+i \gamma_{n}$ and $w_{n}=\beta_{n}+i \delta_{n}$, whence

$$
\left|z_{n}\right|=\left|\bar{z}_{n}\right|=\left|w_{n}\right|=\left|\bar{w}_{n}\right|=1,
$$

and so $z x, \bar{z} x, w x, \bar{w} x$ are all in $E$. Since $E$ is a linear space it follows that $\alpha x$, $\beta x \in E$ and so $\lambda x \in E$. Hence $(3) \rightarrow(1)$.

Theorem 2. In any normed linear space $X$ the statements (4), (5), (6), (7) and (8) are equivalent.

Proof. Let (4) hold, $x \in E$ and $\left\|y_{n}\right\| \leq\left\|x_{n}\right\|$. If $\left\|x_{n}\right\|=0$ we define $\lambda_{n}=1$ and if $\left\|x_{n}\right\|>0$ we define $\lambda_{n}=\left\|y_{n}\right\| /\left\|\mid x_{n}\right\|$, so that in every case $0 \leq \lambda_{n} \leqq 1$ and $\left\|v_{n}\right\|=\left\|\lambda_{n} x_{n}\right\|$. Now define $\mu_{n}$ such that $\lambda_{n}^{2}+\mu_{n}^{2}=1$ and write $z_{n}=\overline{\lambda_{n}}+i \mu_{n}$. Then

$$
\left\|z_{n}, x_{n}\right\|=\left\|\bar{z}_{n} x_{n}\right\|=\left\|\cdot x_{n}\right\|
$$


and so (4) implies that $z x$ and $\bar{z}_{x}$ are in $E$, whence $\lambda x \in E$. Since $\left\|i_{n}\right\|=\left\|\lambda_{n}, x_{n}\right\|$ it follows from (4) that $y \in E$. Herice (4) $-(5)$.

Now let (5) hold, $x \in E$ and $\left\|A_{h}\right\|<M$ for all $n \in N$. Then

$$
\left\|A_{n}\left(M^{-1} \cdot x_{n}\right)\right\| \leq\left\|x_{n}\right\| .
$$

Hence $M^{-1}\left(A_{n} x_{n}\right) \in E$, so $A x \in E$, whence $(5)-(6)$.

It is trivial that $(6) \rightarrow(7) \rightarrow(8)$.

Finally, let (8) hold, $x \in E$ and $\left\|y_{n}\right\|=\left\|x_{n}\right\|$. By the Hahn-Banach theorem there exists $f_{n} \in X^{*}$ with $\left\|f_{n}\right\|=1$ and $f_{n}\left(x_{n}\right)=\left\|x_{n}\right\|$. If $\left\|x_{n}\right\|=0$ we define $A_{n}=I$, the identity operator of $B(X)$, and if $\left\|x_{n}\right\|>0$ we define

$$
A_{n}(w)=f(w) y_{n} /\left\|, x_{n}\right\|
$$

for each $w \in X$. Then. for all $n \in N$, it is clcar that $\left\|A_{n}\right\|=1$ and $y_{n}=A_{n}, x_{n}$. so it follows from (8) that $l=A x \in E$. Hence $(8)-(4)$. which completes the proof.

Theorem 3. In any normed linear space $X$ any one of the statements (4) 10 (8) implies all of the statements (1) w (3). But (I) is equivatem to (4) if and only if $X$ is one-dimensional.

Proof. For the first part of the theorem it is sulficent to show that (8) $\rightarrow$ (3). Let (8) hold, $x \in E$ and $\left|\lambda_{n}\right|=1$. Now define $A_{n} \in B(X)$ by $A_{n}(w)=$ $\lambda_{n} w$ for each $w \in X$. Then $\| A_{n}|=| \lambda_{n} \mid=1$, whence (8) implies that $\lambda x=A x \in E$, so $(8) \rightarrow(3)$.

It is straightforward to verily that if $X$ is one-dimensional then $(1) \rightarrow(4)$.

Finally, suppose $(1) \rightarrow(4)$ but assume that the dimension of $X$ exceeds 1 . Let $\left\{b_{1}, b_{2}, \ldots\right\}$ be a Hamel base for $X$ and let us define $E=s\left(\left[b_{1}\right]\right)$, so that $x \in E$ is of the form $x_{n}=\alpha_{n} b_{1}$ for all $n \in N$. It is clear that (1) holds, whence (4) holds. Now we define, for all $n \in N$,

$$
x_{n}=\left\|b_{2}\right\| b_{1} \text { and } y_{n}=\left\|b_{1}\right\| b_{2} \text {. }
$$

Then $x \in E$ and $\left\|x_{n}\right\|=\left\|y_{n}\right\|$, whence $y \in E$, so $y_{n}=\alpha_{n} b_{1}$. Consequently we have $\alpha_{1} b_{1}=\left\|b_{1}\right\| b_{2}$, which is contrary to the fact that $b_{2}$ are elements of the base. 


\section{THE DELTA DUAL}

Henceforth we assume that $X$ is an abstract non-commutative normed algebra, not necessarily containing an identity element. As before $x=\left(x_{k}\right)$, $l=\left(v_{k}\right)$ denote elements of the space $s(X)$.

For any non-empty subset $E$ of $s(X)$ we now introduce its delta dual $E^{\$}$ defined as follows:

$$
E^{\hat{\delta}}=\left\{v \in s(X): \sum_{k=1}^{\infty}\left(\left\|x_{k} y_{k}\right\|+\left\|y_{k} x_{k}\right\|\right)<\infty \text { for all } x \in E\right\} .
$$

It is immediate that $E^{\delta}$ is a linear subspace of $s(X)$ even though $E$ may not be a linear subspace. Also, it is clear that we have $E \subset E^{8 \delta}$ for any nonempty $E$.

If it happens that $E=E^{\delta \delta}$ we shall define $E$ to be $\delta$-perfect.

Theorem 4. If $E$ is $\delta$-perfect then $E$ is solid in the sense of (1), (2) or (3).

Proof. Let $x \in E,\left|\lambda_{k}\right| \leq 1$ for all $k \in N$ and $y \in E^{\delta}$. Then

$$
\sum\left(\left\|\lambda_{k} \cdot x_{k} y_{k}\right\|+\left\|y_{k} \lambda_{k} \cdot x_{k}\right\|\right) \leq \Sigma\left(\left\|x_{k} y_{k}\right\|+\left\|y_{k} \cdot x_{k}\right\|\right)<\infty,
$$

which implies $\lambda x \in E^{\delta \delta}=E$.

Or course there are solid spaces which are not $\delta$-perfect; for example $E=6$ when $X$ is the complex field $C$.

Next we examine the relation between the space $l_{\infty}(X)$ of bounded sequences and the $\delta$-dual of $I_{1}(X)$. Since

$$
\left\|v_{k}\right\| \leq \sup _{k}\left\|y_{k}\right\|:=\|.\|_{\infty}
$$

for each $y \in I_{\infty}(X)$ we have

$$
\sum_{k=1}^{\infty}\left(\left\|x_{k} y_{k}\right\|+\left\|y_{k}, x_{k}\right\|\right) \leq 2\|y\|_{\infty} \sum_{k=1}^{\infty}\left\|x_{k}\right\|
$$

for each $x \in l_{1}(X)$, whence

$$
l_{x}(X) \subset r_{1}^{S}(X)
$$

for any normed algebra $X$. In case $X$ contains a certain type of element, which we shall call an almost identity, we shall be able to prove in Theorem 5 below that there is equality in (9). 
Given a normed algebra $X$ we say that $X$ has an almost identity $z \in X$ if for such a $z$ there is a positive constant $c$ such that

$$
c\|x\| \leq\|x z\|+\|z x\|, \quad \text { for all } x \in X \text {. }
$$

If $X$ has identity $e$, in the usual sense that $x e=e x=x$ for all $x \in X$, then $e$ is obviously an almost identity. We note also that the normed algebra $c_{0}$ with $x y:=\left(x_{k} y_{k}\right)$ and $\|x\|=\sup _{k}\left|x_{k}\right|$ has no almost identity. For if $z \in c_{0}$ were such an identity then for some positive $c$ we have $c\|x\| \leq 2\|x z\|$ for all $x \in c_{0}$. Choose $n$ with $\left|z_{n}\right|<c / 2$ and let $x=e_{n}$, the $n$-th unit vector in $c_{0}$. Then $c \leq 2\left|z_{n}\right|<c$, a contradiction.

Theorem 5. If $X$ has an almost identit! then $l_{\infty}(X)=l_{1}^{\delta}(X)$.

Proof, In view of (9) we need only show that $f_{1}(X) \subset l_{\infty}(X)$. Let $y \in \ell_{1}^{\delta}(X)$, so that for all $x \in l_{1}(X)$,

$$
\sum_{k=1}^{\infty}\left(\left\|x_{k} y_{k}\right\|+\left\|y_{k} x_{k}\right\|\right)<\infty .
$$

Applying the Banach-Steinhaus theorem, there is a positive constant $M$ such that for all $n \in N$ and all $x \in I_{1}(X)$,

$$
\sum_{k=1}^{n}\left(\left\|x_{k} b_{k}\right\|+\left\|y_{k} x_{k}\right\|\right) \leq M \sum_{k=1}^{\infty}\left\|x_{k}\right\| .
$$

Now take any $n \in N$ and define $x_{n}=z$ and $x_{h}=0$ for $k \neq n$, where $z$ is an almost identity. Hence we have $c\left\|y_{n}\right\| \leq M\|z\|$, which implies that $y \in l_{\infty}(X)$, and the proof is complete.

It is interesting to note in the next theorem that there are normed algebras $X$ without an almost identity such that equality holds in (9).

Theorem 6. $l_{\infty}\left(c_{0}\right)=l_{1}^{\delta}\left(c_{0}\right)$.

Proof. By the argument of Theorem 5 there is a number $M$ such that for all $n \in N$ and all $x \in l_{1}\left(c_{0}\right)$,

$$
2\left\|x_{n}, v_{n}\right\| \leq M \sum_{k=1}^{\infty}\left\|\cdot x_{k}\right\|,
$$


where we take $y \in f_{f}^{f}\left(c_{0}\right)$. Now take any $n \in N$, any $p \in N$ and define $x_{h}=0$ $(k \neq n), x_{n}=e_{p,}$, the $p$-th unit vector in $c_{1 ;}$. Then we have $2\left|r_{n, p}\right| \leq M$, where

$$
\left.b_{n}=\left(y_{n 1}, n_{n 2} \ldots\right) \in c_{n}\right)
$$

for each $n \in N$. Since $n$ and $p$ are arbitrary it follows that $\left\|r_{n}\right\| \leq M / 2$, so $y \in l_{\infty}\left(c_{0}\right)$, as required.

If $E$ is any linear subspace of $s(X)$ then its delta dual generates a natural locally convex topology on $E$ determined by the seminorms

$$
p_{l}(x)=\sum_{h=1}^{\infty}\left(\left\|x_{h} y_{h}\right\|+\left\|r_{k} x_{k}\right\|\right)
$$

for each $x \in E$ and each $y \in E^{\delta}$. We shall call this the $E^{6}$ topology on $E$.

In conclusion we give the following result:

Theorem 7. If the normed algebra $X$ has an almost identity $z$ then the $f_{1}(X)$ topology on $l_{1}(X)$ coincides with the norm topology of $l_{1}(X)$.

Proof. As usual, the norm topology of $l_{1}(X)$ is given by the norm

$$
\|x\|=\sum_{k=1}^{\infty}\left\|x_{k}\right\|
$$

for each $x=\left(x_{k}\right) \in I_{1}(X)$.

First we show that the $f_{1}^{\delta}(X)$ topology is weaker than the norm topology of $l_{1}(X)$ even when $X$ has no almost identity. Let $\epsilon>0$ and $y_{1}, y_{2}, \ldots, y_{r} \in l_{1}^{\delta}(X)$. where

$$
y_{i}=\left(y_{i 1}, y_{i 2}, \ldots\right)
$$

By the argument of Theorem 5 there are positive numbers $M_{1}, \ldots, M_{r}$ such that

$$
p_{r_{i}}(x) \leq M_{i}\|x\|
$$

for $i=1,2 \ldots r$ and for all $x \in l_{1}(X)$. Taking $M$ to be the largest of the $M_{i}$ it follows that if $\|x\|<\epsilon / M$ then

$$
\sup \left\{p_{r,}(x): i=1,2, \ldots, r\right\}<\epsilon,
$$


whence, in the usual notation, the sphere $S(0, \epsilon / M)$ is contained in the neighbourhood

$$
U\left(0, p_{y_{1}}, p_{r_{2}}, \ldots, p_{y_{r}}, \epsilon\right) .
$$

Conversely, suppose that $X$ has an almost identity $z$ with corresponding constant $c$, and let $\epsilon>0$ be given. If we define

$$
y=(z, z, z, \ldots)
$$

then $y \in f_{1}^{\delta}(X)$ by 'Theorem 5 . Hence if $x \in U\left(0, p_{y}, c \epsilon\right)$ then

$$
\sum_{k=1}^{\infty}\left(\left\|x_{k} z\right\|+\left\|z x_{k}\right\|\right)<c \epsilon
$$

and since $c\left\|x_{k}\right\| \leq\left\|x_{k} z\right\|+\left\|z x_{k}\right\|$ for all $k \in N$ it follows that $\|x\|<\epsilon$, so that $x \in S(0, \epsilon)$, and the proof is complete.

\section{References}

[1] CoOkl: R. G., Infinite matrices and sequence spaces, Macmillan and Co., London, 1949.

[2] Gari.ing, D. J. H., The $\beta$ - and $\gamma$-duality of sequence spaces, Proc. Camb. Phil. Soc., 63 (1967). 963-981.

[3] KörHis. G., Topological Vector Spaces I (English translation by D.I.H. Garling of Topologische lineare Räume I, 1966), Springer-Verlag, 1969. .

[4] KÖTHF, G., and TOEPIITZ, O., Lineare Räume mit unertdlichvielen Koordinaten twh Ringe unendlicher Marrizen, J.f. reine u. angew. Math.. /7/ (1934), 193-226.

[5] MAmıox. I. J., Infinite marrices of operators, Springer-Verlag. Berlin, 1980.

Jeparment ol Pure Mathematics

Queen's University of Belfiatst

Beliast BJ'7 INN

Unied Kingrdorn

Recibido: 29 de mayo de 1990 